\title{
Síndrome de Parsonage-Turner en infancia y adolescencia. Caso clínico
}

\author{
Parsonage-Turner syndrome in childhood and adolescence. Case report
}

\author{
Dra. Sagrario Pérez-de la Cruz
}

\begin{abstract}
RESUMEN
Se presenta el caso de un varón de 17 años con diagnóstico de síndrome de Parsonage-Turner recidivante en brazo derecho. En su historial clínico se diagnosticó neuralgia amiotrófica de miembro superior en tres ocasiones anteriores, con clínica semejante en cada uno de los episodios, aunque alternándose el miembro superior afecto. Estuvo en tratamiento en los Servicios de Rehabilitación de dos centros hospitalarios. A la exploración física en cada recidiva presenta dolor intenso en el hombro y brazo, y pérdida de fuerza en escápula y miembro superior afecto. El objetivo de su tratamiento combina tratamiento del dolor $\mathrm{y}$, junto con fisioterapia, luchar contra la atrofia muscular que presenta.

El paciente evolucionó favorablemente en cada uno de los episodios que presentó y, en la actualidad, se muestra asintomático y dado de alta en el servicio de Rehabilitación.

Palabras clave: sindrome de Parsonage-Turner, neuralgia, rehabilitación.
\end{abstract}

\section{SUMMARY}

We present the case of a 17-year-old male whose diagnosis is Parsonage-Turner syndrome relapsing in the right arm. In his medical record, he was diagnosed as having amyotrophic neuralgia of the upper limb in three previous occasions. The diagnosis was similar in all episodes, although the affected upper limb was alternating. He was treated in the Rehabilitation Services of two hospitals. At physical examination, in every relapse, he showed acute pain in both, shoulder and arm, and loss of strength in the shoulder blade and the affected upper limb. The aim of his treatment combines the healing of the pain and, together with physiotherapy, fighting against muscular atrophy.

The patient evolved favourably in each of the episodes. Nowadays, he does not show any symptom, and he has been discharged from the rehabilitation service.

Key words: Parsonage-Turner syndrome, neuralgia, rehabilitation.

http:/ /dx.doi.org/10.5546/aap.2012.e107

a. Doctora en Fisioterapia. Facultad de Ciencias de la Salud. Universidad de Almería. España.

Correspondencia:

Dra. Sagrario Pérez-de la Cruz: spd205@ual.es

Conflicto de intereses: Ninguno que declarar.

Recibido: 21-3-2012

Aceptado: 1-6-2012

\section{INTRODUCCIÓN}

El síndrome de Parsonage-Turner, o neuralgia amiotrófica es una neuropatía infrecuente que se manifiesta una rápida aparición de dolor intenso y agudo en el hombro, seguido por una debilidad repentina y atrofia progresiva de los músculos inervados por $\mathrm{C} 5-\mathrm{C} 6$ o de otras de raíces nerviosas próximas. ${ }^{1,2}$

La causa exacta del proceso es desconocida hasta la fecha. ${ }^{3,4}$ Los pacientes refieren dolor local del hombro como síntoma más habitual. ${ }^{3}$ Es con frecuencia de inicio súbito y a menudo grave, pudiendo despertar al paciente durante el descanso nocturno. Empeora progresivamente en horas o días y su duración más habitual es de pocas horas a tres semanas; igualmente, suele desaparecer de forma rápida, aunque en ocasiones persiste un leve dolor residual. El tiempo que transcurre hasta la aparición de la paresia es altamente variable y ésta persiste una vez desaparecido el dolor; en un $25 \%$ de los casos es bilateral. ${ }^{5}$ La parálisis es fláccida, en parches y normalmente progresiva; afecta sobre todo a la elevación del hombro y la abducción del miembro superior. Se trata de una afectación de tipo motoneurona inferior, con reflejos hipoactivos y ocasionalmente fasciculaciones. ${ }^{6}$

Atendiendo al músculo afectado, el descrito con más frecuencia es el deltoides, seguido del supraespinoso, infraespinoso, serrato mayor, bíceps, tríceps y extensores del carpo y dedos..$^{6-8}$ Normalmente se trata de un proceso ipsolateral, aunque se describe la afectación bilateral, pero asimétrica en cuanto a los músculos y al grado de afectación.

\section{CASO CLÍNICO}

Varón de 17 años que acude al servicio de rehabilitación del centro de salud de Bolaños de Calatrava (Ciudad Real) España, con el siguiente historial clínico: a los cuatro años de edad fue diagnosticado de neuralgia amiotrófica del hombro izquierdo, con afectación de C5 a C8. En la entrevista inicial sus tutores comentaron que dos meses antes de comenzar el primer brote sufrió una fractura supracondílea de la paleta humeral izquierda sin desplazamiento. Tras años sin presentar signos, apareció una reagudización de 
la patología en el brazo derecho (año 2007). Su diagnóstico fue neuritis idiopática amiotrófica recidivante. La clínica presente fue un cuadro de dolor muy agudo, por lo que fue derivado a un centro hospitalario para su tratamiento. La evolución clínica fue favorable, con desaparición de la sintomatología. Pero a los 16 años, tras permanecer cinco años asintomático, presenta recaída, con afectación en el brazo izquierdo y pérdida de fuerza en deltoides y extensores de muñeca. El tratamiento pautado es deflazacort, tryptizol, trileptal, con posterioridad gammaglobulinas IV durante cuatro días. Presenta igualmente mejoría clínica.

Tras dos meses de evolución de este último episodio, inicia nuevo brote en brazo derecho, con dolor intenso en el hombro y pérdida de fuerza en escápula. Su diagnóstico actual sería compatible con lesión de los nervios radial y axilar derechos, probablemente a nivel del cordón posterior del plexo braquial derecho, en grado de axonotmesis grave para el nervio radial y axonotmesis moderada para el axilar (ver Figura 1).

\section{DISCUSIÓN}

El síndrome de Parsonage-Turner es una alteración de etiología desconocida (con posibles factores desencadenantes) que se caracteriza por un dolor agudo e intenso del hombro y de la extremidad superior, seguida de paresia y atrofia

FIGURA 1. Imagen posterior. Atrofia de la cintura escapular derecha.

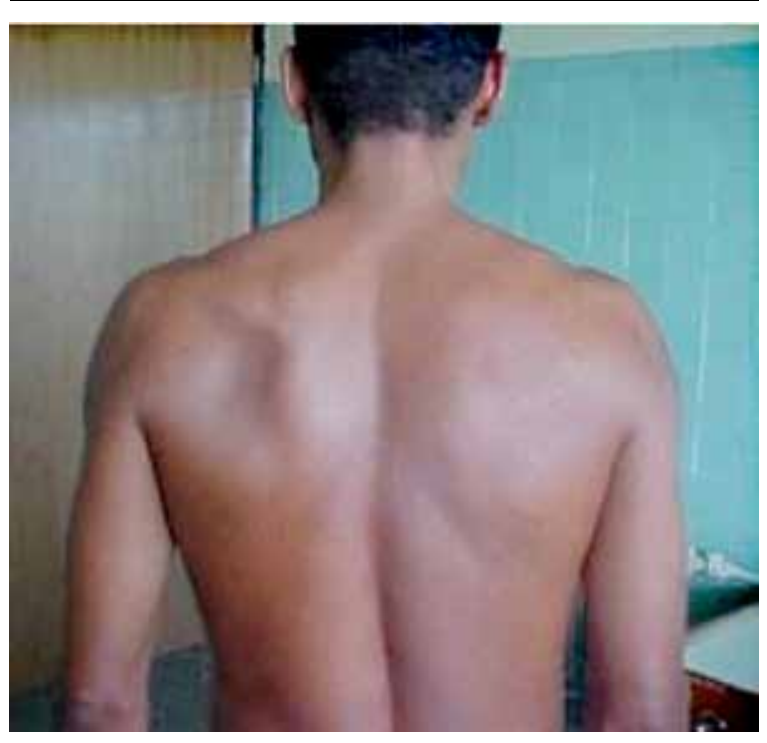

muscular. ${ }^{9}$ La etiología es desconocida, aunque la hipótesis actual es que los episodios son causados por una respuesta mediada por mecanismos inmunitarios al plexo braquial. Se han propuesto una serie de factores precipitantes, como:

- Infecciones, tanto bacterianas (Borrelia) como virales (gripe, VIH). De hecho, Tsairis registró en su estudio que, en el $25 \%$ de los pacientes, el inicio de los síntomas era precedido por una infección viral sistémica. ${ }^{4}$

- Patología de causa monogénica con un patrón de herencia autosómico dominante (la/s anomalía/s generalmente aparecen en cada generación. Cada niño afectado de un padre afectado tiene un $50 \%$ de probabilidades de heredar la enfermedad).

- Se ha intentado relacionar con situaciones de postinmunización (vacunas), y de autoinmunidad. ${ }^{10,11}$

- También con posibles causas asociadas con los traumatismos, o el ejercicio físico extenuante.

No obstante, el papel de todos estos factores permanece todavía en período de comprobación.

Los nervios más afectados son el axilar, el supraescapular, el torácico y el musculocutáneo. Esta neuritis tiene un buen pronóstico a largo plazo; sin embargo, el tiempo que demanda la resolución completa es variable. Dentro de los factores de lentitud en la recuperación está la intensidad y duración de los síntomas, número de nervios afectados y el grado de compromiso del plexo braquial. ${ }^{9}$

No existe un tratamiento específico para el síndrome de Parsonage-Turner, ${ }^{10}$ pero existe unanimidad en cuanto a que debe ser conservador y no quirúrgico. Los objetivos terapéuticos son reducir el dolor, prevenir la restricción de la movilidad y recuperar la fuerza muscular. ${ }^{12}$

En las fases iniciales se ha recomendado tratamiento con corticoides e inmunoglobulinas, basándose en la hipótesis neuroalérgica del síndrome, ${ }^{13}$ con el objeto de prevenir la debilidad muscular, pero la eficacia de estas medidas no ha sido probada. Van Alfen et al. ${ }^{14}$ describen 41 pacientes tratados con corticoides en una serie de 246 pacientes. El tiempo promedio transcurrido hasta el inicio del tratamiento fue de 13 días. En 18 pacientes, se utilizó el mismo esquema terapéutico consistente en un tratamiento de 2 semanas de prednisolona oral, $60 \mathrm{mg}$ diarios en la primera semana y reducción con $10 \mathrm{mg}$ por día en la segunda semana; en los otros pacientes, variaron la dosis y la duración. Cuatro de 20 pacientes 
(20\%) manifestaron subjetivamente que el tratamiento había tenido una influencia positiva en sus síntomas. El tiempo promedio transcurrido hasta obtener una disminución del dolor fue de 5 días, en comparación con 20 días en el grupo sin tratar. En el 8\% de los pacientes tratados se documentó una recuperación total en un plazo de un mes desde la aparición, en comparación con el 5\% de los pacientes en el grupo sin tratar.

Tras la fase aguda, el tratamiento es fundamentalmente rehabilitador, con los objetivos de mantener la movilidad articular completa y estimular la contracción muscular. Los ejercicios de movilidad activa y pasiva deben comenzarse tan pronto como se haya conseguido el adecuado control del dolor. Pueden estar indicados los ejercicios de fortalecimiento de la musculatura del manguito de los rotadores y de estabilización escapular. El momento y el papel de fortalecimiento de ejercicios dependen del grado de denervación muscular, del grado de debilidad y del grado de alteración de la biomecánica y del nivel premórbido funcional de actividad para ese paciente. Los ejercicios de fortalecimiento no son indicados en los músculos denervados y el papel de la estimulación eléctrica es objeto de controversia, pero debe ser considerado cuando el estado de denervación se prolonga más de cuatro meses. En estos casos, la regeneración axonal y la reinervación muscular puede ser retardada. La electromiografía se usa como prueba de seguimiento de los músculos involucrados para demostrar el grado de reinervación. Esta prueba es útil para ayudar a determinar cuándo los músculos pueden tolerar un fortalecimiento más intensivo.

En los casos excepcionales en los que no se consigue una buena recuperación física al cabo del segundo año, puede considerase la opción de la cirugía, con el objeto de mejorar la abducción del hombro. Se han comunicado algunos casos de injerto de nervio, transferencia de tendón, así como fijación quirúrgica de la escápula al tórax. ${ }^{15}$

Como conclusión, corresponde destacar que el síndrome de Parsonage-Turner es una patología poco frecuente, pero potencialmente grave, don- de es fundamental un buen y rápido diagnóstico clínico, con las tecnologías diagnósticas actuales, y que para optimizar el tratamiento es necesario un abordaje multidisciplinario, que incluya la valoración y el tratamiento conjunto de los servicios de Neurología, Rehabilitación, Fisioterapia y Terapia ocupacional.

\section{BIBLIOGRAFÍA}

1. Hyde GP, Postma GN, Caress JB. Laryngeal paresis as a presenting feature of idiopathic brachial plexopathy. Otolaryngol Head Surg 2001;124:575-6.

2. Tsao BE, Ostrovskiy DA, Wilbourn AJ, Shields RW. Phrenic neuropathy due to neuralgic amyotrophy. Neurology 2006;66:1582-4.

3. Kuhlenbaumer G, Meuleman J, De Jonghe P, Falk B, et al. Hereditary neuralgic amyotrophy (HNA) is genetically heterogeneus. J Neurol 2001;248:861-5.

4. Tsairis P, Dyck PJ, Mulder DW. Natural history of brachial plexus neuropathy. Report on 99 patients. Arch Neurol 1972;27:109-17.

5. González Reimers E, Ruiz Lacambra J, López García J, Rodríguez Gaspar M, et al. Dolor y amiotrofia en miembros superiores en una paciente cirrótica afecta de Mal de Pott. An Med Interna 2005;22:79-81.

6. Raba Oruña S, Pérez Martín A, López Lanza JR, Guijarro Bezanilla M, et al. Síndrome de Parsonage-Turner: raro pero existe. Dialnet 2005;78:664-5.

7. Briceño F, Rodríguez S. Plexopatía braquial: idiomática vs. traumática. Reumatol Clin 2009;5:165-95.

8. Aymond JK, Goldner JL, Hardaker WT. Neuralgic amyotrophy. Orthop Rev 1989;18:1275-9.

9. Muse L, Contreras O. Síndrome de Parsonage-Turner o neuritis braquial: a propósito de dos casos clínicos. Rev Chil Radiol 2003;9:137-9.

10. Danachet $Y$, Besson G. Syndrome de Parsonage et Turner et artérite de Horton. Rev Neurol (Paris) 2002;158:354-6.

11. Vanermen B, Aetgerts M, Hoogamartens M, Fabry G. The syndrome of Parsonage-Turner. Discussion of clinical features with a review of 8 cases. Acta Orthop Belg 1991; 57:414-9.

12. Mamula C, Erhard RE, Piva SR. Cervical radiculopathy or Parsonage-Turner syndrome: differential diagnosis of a patient with neck and upper extremity symptoms. J Orthop Sports Phys Ther 2005;35:659-64.

13. Fink GR, Haupt WF. Neuralgic amyotrophy (ParsonageTurner syndrome) following streptokinase thrombolytic therapy. Dtsch Med Wochenschr 1995;120:959-62.

14. Van Alfen N, van Engelen B. The clinical spectrum of neuralgic amyotrophy in 246 cases. Brain 2006;129:438-50.

15. Conway RR. Neuralgic amyotrohy: uncommon but not rare. Mo Med 2008;105:168-9. 